\title{
General brane cosmologies and their global spacetime structure
}

\author{
Peter Bowcock*, Christos Charmousis ${ }^{\dagger}$, Ruth Gregory. ${ }^{\ddagger}$ \\ Centre for Particle Theory, Durham University, South Road, Durham, DH1 3LE, U.K.
}

(October 22, 2018)

\begin{abstract}
Starting from a completely general standpoint, we find the most general brane-Universe solutions for a three-brane in a five dimensional spacetime. The brane can border regions of spacetime with or without a cosmological constant. Making no assumptions other than the usual cosmological symmetries of the metric, we prove that the equations of motion form an integrable system, and find the exact solution. The cosmology is indeed a boundary of a (class II) Schwarzschild-AdS spacetime, or a Minkowski (class I) spacetime. We analyse the various cosmological trajectories focusing particularly on those bordering vacuum spacetimes. We find, not surprisingly, that not all cosmologies are compatible with an asymptotically flat spacetime branch. We comment on the role of the radion in this picture.
\end{abstract}

PACS numbers: 04.50.+h, 11.25.Mj, 98.80.-k hep-th/0007177

*E-mail address: Peter.Bowcock@durham.ac.uk

${ }^{\dagger}$ E-mail address: Christos.Charmousis@durham.ac.uk

$\ddagger$ E-mail address: R.A.W.Gregory@durham.ac.uk 


\section{INTRODUCTION}

The idea that our four-dimensional universe might in fact, upon closer inspection, be higher dimensional has always been compelling, although it has been conventionally supposed that the extra dimensions are small and compact. An almost diametrically opposed view, wherein the extra dimensions are large and noncompact [1] has gained ground recently, in which our universe is a 'defect' (see [2] for early work) in an anti-de Sitter bulk - the negative cosmological constant providing an effective or exotic compactification [3] of the spacetime. In most models, the universe is taken to be a domain wall, (i.e. of codimension one - although models of higher codimension have been considered [4]) since this is most naturally supported by heterotic string theory [5] []. The original models of Randall and Sundrum |1 focussed on the main features of having either one or two domain wall universes at orbifold fixed points of a single extra dimension; in the case of the two-wall model the second wall had negative energy and tension. The compelling feature of these brane-world models is that gravity has a four-dimensional character on the universe-domain-wall with short-range five-dimensional corrections coming from Kaluza-Klein (KK) modes. Indeed, it was demonstrated explicitly by Garriga and Tanaka [9] that linearized gravity on the brane was indeed Einstein gravity for a single wall universe (see also [10]). The key feature of the Randall Sundrum (RS) (and indeed many other) models is that the induced metric on the wall (or walls) is flat Minkowski spacetime. In general, a domain wall spacetime is not static, but has a de-Sitter expansion along its spatial directions, however in the presence of a bulk cosmological constant, the effect of the wall energy and tension is neutralized, and a static solution is allowed. However, our universe is not Minkowski spacetime, nor is it a localized linearized perturbation thereof. Therefore, if brane universe models are to be relevant, cosmological brane solutions are the obvious next step.

A general homogeneous and isotropic brane cosmology is simply a wall with energy, e, and tension, $\mathrm{T}$, which are no longer fixed by the special domain wall relation $\mathrm{E}=\mathrm{T}$. For example, we might wish to set

$$
\mathrm{E}=\mathrm{E}_{0}+\rho \quad ; \quad \mathrm{T}=\mathrm{T}_{0}-p
$$

and then take $\rho$ and $p$ to be our 'cosmological' energy density and pressure respectively. This is what is conventionally done, with $\rho$ and $p$ obeying an equation of state (rather than $\mathrm{E}$ and $\mathrm{T}$ ), although it should be noted that when $\rho$ and $\mathrm{E}$ are of a similar order of magnitude, there is no reason to suppose that the equation of state will continue to hold with this choice of $\rho$ and $p$, which become rather arbitrary at that point.

Much of the work on brane-cosmological models to date has taken a 'brane-based' ap-

proach, notably the work of Binetruy et. al. [11,12] (see also [13]), in which the RS spacetime is generalized to allow for time-dependent cosmological expansion. However, Binetruy et. al. were concerned primarily with deriving the four-dimensional brane-cosmological equations, and for simplicity when finding explicit solutions, chose a Gaussian Normal gauge in which $g_{n n}$, the component of the metric normal to the wall, was set to unity. Therefore, while the brane cosmology was readily apparent in their approach, the bulk spacetime structure was less transparent, with coordinate singularities indicating the breakdown of the rather restrictive GN gauge. A more general brane-based approach is epitomized by the work of Shiromizu et. al. [14] (see also Maartens, [15], for an excellent brane-based description of 
bulk effects) in which the Gauss-Codazzi formalism is used to obtain a pure-brane 'Einstein' equation, in which the bulk geometry is encoded in a single tensor $\mathcal{E}_{\mu \nu}$. While such a brane-based approach has the attraction of being a simple generalization of the standard four-dimensional FRW equations, the effect of the bulk on the brane is somewhat less transparent, with the tensor $\mathcal{E}_{\mu \nu}$ hiding a multitude of sins.

An alternate approach for deriving cosmological solutions was taken by Ida [16], who instead considered a 'bulk-based' point of view, in which the most general static AdS solution with the appropriate symmetries:

$$
d s^{2}=h(r) d t^{2}-h^{-1}(r) d r^{2}-r^{2}\left[\frac{d \chi^{2}}{1-\kappa \chi^{2}}+\chi^{2} d \Omega_{I I}^{2}\right]
$$

(where $h(r)=\kappa-\frac{\mu}{r^{2}}+k^{2} r^{2}$ ) is taken, and the brane becomes an arbitrary boundary of two versions of this spacetime, with possibly differing masses on each side. The IsraelGauss-Codazzi equations then give the energy and tension of the boundary as a function of its trajectory. The beauty of this approach is that it is quite general and does not rely on any $Z_{2}$ symmetry around the wall itself; it is also straightforward to derive branecosmological evolution equations - the cosmological solution then becomes a wall moving in AdS spacetime; the only disadvantage is that it is perhaps a little more abstract than the brane-based approach. A coordinate transformation relating the solutions of [11, 12] to Ida's solutions was found by by Mukohyama et. al. [17]. Ida's work in fact generalizes the work of Kraus [18] (see also [19] for related string theoretic work, and [20], for an AdS/CFT perspective on the issue), who derived the most general $Z_{2}$ symmetric domain wall solutions by taking slicings of (2), and so can apply to models with different cosmological constants such as the Lykken-Randall (LR) model, [21], for example.

What we aim to do in this paper is to bridge the gap between the 'brane-based' approach, where the brane represents a fixed boundary in some time-evolving spacetime, and the 'bulkbased' approach, where the wall (or spacetime boundary) follows some timelike trajectory in a static bulk spacetime. We start off by considering the most general brane-based formalism, following the approach of Ipser and Sikivie [22], finding the general wall solutions for a fixed wall of constant spatial curvature embedded in a bulk of constant curvature. We then demonstrate how a fixed wall embedded in a non-static spacetime is strictly equivalent to a moving wall embedded in a static spacetime thereby establishing in full generality the equivalence between the two approaches. This is true for a wall of completely arbitrary equation of state separating spacetimes which may even have a different cosmological constant. Having shown this equivalence we then proceed to find and study the most general wall trajectories, i.e. cosmological evolution equations, in a static AdS or even flat background. The reason for studying such a general set-up is that some more recent generalizations of the RS scenario have involved not only non $Z_{2}$ symmetric walls, such as the 'Millenium Model' of Kogan et. al. [23] consisting of two positive tension walls at orbifold fixed points with an additional negative tension wall freely moving inbetween, but also patching together of spacetimes with different cosmological constant, such as the GRS model [24], in which the central $Z_{2}$ symmetric wall is flanked by two negative tension branes with flat space in the exterior. The curious feature of these apparently contrived models is that gravity not only changes nature at short but also at ultra-large scales, becoming weaker for the Millenium model, and five-dimensional for the GRS model. (In fact gravity for the GRS model has 
several peculiarities, [25 27], which may be ameliorated by a hybrid double-wall variant of the Millenium model, [28], in which the negative tension wall of the Millenium model is replaced by two negative tension walls with a slice of flat space inbetween.)

While we might expect that cosmology in such models is potentially delicate, the beauty of the geometrical approach is that the search for a cosmology becomes a local question of finding a suitable trajectory for the spacetime boundaries, thus the presence of extra walls is irrelevant - unless these walls collide. We therefore do not consider issues such as radion stabilization [29], or the like, simply examining the possible trajectories (and hence cosmological solutions) of the brane universes.

In the next section we set up our formalism and then find the general solution to the Einstein equations for a constant spatial curvature wall in a constant curvature spacetime, generalizing Taub's solutions [30] to allow for the presence of a cosmological constant in the bulk. We then show how from this brane-based approach we can cross over to the bulkbased approach for a wall of arbitrary trajectory evolving in a static spacetime. In section III we establish the most general cosmological evolution equations for the different classes of spacetime solutions. In the following section we analyze some specific cases of interest in cosmology finding the wall trajectories analytically. We make some concluding remarks in Section V.

\section{GENERAL WALL SPACETIMES}

In this section we derive the general spacetime of a brane universe, and the equations of motion it must obey. As per usual, we shall be modelling our four-dimensional Universe, $\mathcal{U}$, to be an infinitesimally thin wall type defect of constant spatial curvature embedded in a five-dimensional spacetime. In other words, we are looking for a spacetime with planar (or spherical/hyperboloidal) symmetry in three of its spatial directions, which has one (or more) hypersurfaces on which the spacetime curvature has a distributional singularity corresponding to a $\delta$-function source for the wall energy-momentum. The most general metric admitting this symmetry can be written in the form

$$
d s^{2}=e^{2 \nu(\mathrm{t}, \mathrm{z})}(B(\mathrm{t}, \mathrm{z}))^{-2 / 3}\left(d \mathrm{t}^{2}-d \mathbf{z}^{2}\right)-B^{2 / 3}\left[\frac{d \chi^{2}}{1-\kappa \chi^{2}}+\chi^{2} d \Omega_{I I}^{2}\right]
$$

where $\kappa=0, \pm 1$ represents the spatial curvature of the 3 -spatial sections, and $\nu, B$ will satisfy the bulk Einstein equations (with or without a cosmological constant), as well as appropriate jump conditions at the wall which we will choose to set at $\mathbf{z}=0$.

We start by summarizing the jump conditions for later use. As is conventional, we denote the normal to $\mathcal{U}$ by $n_{a}$, in terms of which the first fundamental form, $h_{a b}$, of the wall is given by

$$
h_{a b}=g_{a b}+n_{a} n_{b}
$$

which is simply the projection of the bulk metric on $\mathcal{U}$. The second fundamental form, or extrinsic curvature of $\mathcal{U}$ is

$$
K_{a b}=h_{(a}^{c} h_{b)}^{d} \nabla_{c} n_{d}
$$


The intrinsic and extrinsic geometry of $\mathcal{U}$ are related to the bulk curvature via the Gauss Codazzi equations

$$
\begin{aligned}
R_{a c b d} n^{c} n^{d}=K_{a c} K_{b}^{c}-\mathcal{L}_{n} K_{a b} & =R_{a b}^{(4)}-R_{c d} h_{a}^{c} h_{b}^{d}-K_{a c} K_{b}^{c}+K K_{a b} \\
h^{b c} \nabla_{b} K_{c a}-h_{a}^{b} \nabla_{b} K & =R_{b c}^{(4)} h_{a}^{b} n^{c} \\
R^{(4)}-K_{a b} K^{a b}+K^{2} & =2 G_{a b} n^{a} n^{b}
\end{aligned}
$$

where $R_{a b}^{(4)}$ is the intrinsic Ricci tensor obtained from $h_{a b}$, and $\mathcal{L}_{n}$ is the Lie derivative with respect to $n_{a}$.

Since the wall's energy-momentum tensor $T_{a b}^{w}$ is a distributional source, we can now integrate (6a) across the wall, and defining

$$
S_{a b}=\int T_{a b}^{w} d l
$$

we immediately arrive at the Israel junction conditions [31

$$
\Delta K_{a b}=\left[S_{a b}-\frac{1}{3} S h_{a b}\right]
$$

where $\Delta K_{a b}=K_{a b}^{+}-K_{a b}^{-}$is the jump in the extrinsic curvature, and we have set $8 \pi G_{5}=1$.

Furthermore taking the sum and difference of (6a) and (6b) respectively and using (8) we have,

$$
\begin{aligned}
R^{(4)}-\bar{K}_{a b} \bar{K}^{a b}+\bar{K}^{2} & =\frac{1}{4}\left(S_{a b} S^{a b}-\frac{1}{3} S^{2}\right)+2 \bar{G}_{n n} \\
\bar{K}_{a b} S^{a b} & =2 \Delta G_{n n} \\
\nabla_{b}^{(4)} S^{b c} & =0 \\
\nabla_{b}^{(4)} \bar{K}_{a}^{b}-\nabla_{a}^{(4)} \bar{K} & =0
\end{aligned}
$$

where $\bar{Q}=\left(Q^{+}+Q^{-}\right) / 2$ stands for the mean of a quantity across the wall. We should stress that (9) are in fact integrability conditions and hence will result from the Einstein and junction conditions (8).

Since we are looking for cosmological solutions, we shall assume that our brane Universe is made of homogeneous and isotropic matter, hence

$$
S_{a b}=\mathrm{E} u^{a} u^{b}+\mathrm{T}\left(h^{a b}-u^{a} u^{b}\right)
$$

where $\mathrm{E}$ is the surface energy density and $\mathrm{s}$ the tension of the brane $\mathcal{U}$. The timelike vector $u^{a}$ is the 5-velocity of an observer comoving with the brane Universe. Obviously if $\mathrm{E}=\mathrm{T}$ we have a domain wall, and if $\mathrm{T}=0$, a dust wall.

We begin by finding the most general bulk solution before examining these boundary junction conditions. The bulk Einstein equations in this case are simply,

$$
R_{a b}=-\frac{2}{3} \Lambda g_{a b}
$$

where $\Lambda$ is the cosmological constant. For the metric (3) these give the following system of partial differential equations, 


$$
\begin{aligned}
B_{, \mathrm{tt}}-B_{, \mathbf{z z}} & =\left(2 \Lambda B^{1 / 3}-6 \kappa B^{-1 / 3}\right) e^{2 \nu} \\
\nu_{, \mathrm{tt}}-\nu_{, \mathbf{z z}} & =\left(\frac{\Lambda}{3} B^{-2 / 3}+\kappa B^{-4 / 3}\right) e^{2 \nu} \\
\nu_{, \mathbf{z}} B_{, \mathrm{t}}+\nu_{, \mathrm{t}} B_{, \mathbf{z}} & =B_{, \mathrm{tz}} \\
2 \nu_{, \mathbf{z}} B_{, \mathbf{z}}+2 \nu_{, \mathrm{t}} B_{, \mathrm{t}} & =B_{, \mathrm{tt}}+B_{, \mathbf{z z}}
\end{aligned}
$$

The particular way of writing the metric (3) shows the Liouville like character of the system of equations, where (12a), (12b) are in the presence of $\Lambda$, non-homogeneous coupled wave equations and (12d) and (12d]) will turn out to be integrability conditions. This is not too surprising since we are effectively studying a $1+1$ gravity problem by considering the Kaluza-Klein reduction of the constant curvature spacelike dimensions.

It proves easiest to rewrite (12) in light-cone coordinates,

$$
u=\frac{\mathrm{t}-\mathrm{z}}{2}, \quad v=\frac{\mathrm{t}+\mathrm{z}}{2}
$$

in which

$$
\begin{aligned}
B_{, u v} & =\left(2 \Lambda B^{1 / 3}-6 \kappa B^{-1 / 3}\right) e^{2 \nu} \\
\nu_{, u v} & =\left(\frac{\Lambda}{3} B^{-2 / 3}+\kappa B^{-4 / 3}\right) e^{2 \nu} \\
B_{, u}\left[\ln \left(B_{, u}\right)\right]_{, u} & =2 \nu_{, u} B_{, u} \\
B_{, v}\left[\ln \left(B_{, v}\right)\right]_{, v} & =2 \nu_{, v} B_{, v}
\end{aligned}
$$

First note that $(140,14 \mathrm{~d})$ can be directly integrated to give

$$
e^{2 \nu}=V^{\prime}(v) B_{, u}=U^{\prime}(u) B_{, v}
$$

where $V^{\prime}(v), U^{\prime}(u)$ are arbitrary nonzero functions of $u, v$ for generic $\Lambda, \kappa$. (If $\Lambda=\kappa=0$, then it is possible for one of $U^{\prime}(u)$ or $V^{\prime}(v)$ to vanish - see below.) It is straightforward to see then that $B$ and $\nu$ have the form (where we remind the reader that primes denote ordinary differentiation with respect to the unique variable of the function)

$$
B=B(U(u)+V(v)), \quad e^{2 \nu}=B^{\prime} U^{\prime} V^{\prime}
$$

which reduces the remaining PDE (14a) to the ODE

$$
\begin{aligned}
B^{\prime \prime}-\left(2 \Lambda B^{1 / 3}-6 \kappa B^{-1 / 3}\right) B^{\prime} & =0 \\
\Rightarrow \quad B^{\prime}-\frac{3}{2} \Lambda B^{4 / 3}+9 \kappa B^{2 / 3} & =9 \mu
\end{aligned}
$$

where $\mu$ is an integration constant. This last relation can be integrated to give

$$
\frac{1}{\sqrt{9 \kappa^{2}-3 \mu \Lambda}}\left[\left|r_{-}\right| \tan ^{-1} \frac{r}{\left|r_{-}\right|}-r_{+} \operatorname{coth}^{-1} \frac{r}{r_{+}}\right]=(U+V)
$$

where $r=B^{1 / 3}, c$ is a constant of integration, and 


$$
r_{ \pm}^{2}=\frac{3 \kappa}{\Lambda} \pm \sqrt{\frac{9 \kappa^{2}}{\Lambda^{2}}-\frac{6 \mu}{\Lambda}} .
$$

Hence the general solution to (11) is given by,

$$
d s^{2}=B^{\prime} U^{\prime} V^{\prime} B^{-2 / 3}\left(d \mathrm{t}^{2}-d z^{2}\right)-B^{2 / 3} d x_{I I I}^{2}
$$

where $B=B(U+V)$ satisfies (17) or (18), $U(u)$ and $V(v)$ are arbitrary functions and $d x_{I I I}^{2}$ stands for the three dimensional constant curvature metric written out explicitly in (3).

When $\Lambda=\kappa=0$, (17) gives (wlog) $B=U+V$, and there are now in fact two allowed classes of solution, in the terminology of Taub [30]; the above, (20), being a class II solution. The class I solutions are distinguished by having $U^{\prime}=0$ or $V^{\prime}=0$, but not both, in which case the general metrics are

$$
\begin{aligned}
& d s^{2}=\frac{U^{\prime} H(v)}{U^{2 / 3}}\left(d \mathrm{t}^{2}-d \mathbf{z}^{2}\right)-U^{2 / 3} d \mathbf{x}^{2} \\
& d s^{2}=\frac{V^{\prime} K(u)}{V^{2 / 3}}\left(d \mathrm{t}^{2}-d \mathbf{z}^{2}\right)-V^{2 / 3} d \mathbf{x}^{2}
\end{aligned}
$$

for $U^{\prime} \neq 0$ and $V^{\prime} \neq 0$ respectively. In fact these class I solutions are flat, as we will see in the next section. In summary Einstein's equations (12) admit two distinct classes of solutions I, (21a), and II, (20), which depend on two arbitrary functions.

Having derived the general bulk solutions, now let us examine the constraints, or boundary conditions at $\mathbf{z}=0$ imposed by the wall. For what follows we shall assume $Z_{2}$ symmetry in the $\mathbf{z}$-direction to make notation easier and results more transparent. We shall however be dropping this assumption when actually looking for general cosmological solutions in the next section.

Reflection symmetry about $z=0$ permits us to consider only $z>0$ in $(\sqrt[20]{)}$ since the metric is an even function with respect to $z$. Extrinsic curvature components are thus odd functions in $z$ and hence,

$$
\Delta K_{a b}=2 K_{a b}^{+}, \quad \overline{K_{a b}}=0
$$

In the coordinate system adapted to the wall the intrinsic and extrinsic geometric quantities are greatly simplified, and take the form

$$
\begin{aligned}
\mathrm{E} & =-2 e^{-\nu} B^{-2 / 3} \partial_{z} B=-e^{-\nu} \frac{B^{\prime}\left(V^{\prime}-U^{\prime}\right)}{B^{2 / 3}} \\
\left(\frac{2}{3} \mathrm{E}-\mathrm{T}\right) & =-2 \partial_{z}\left(B^{1 / 3} e^{-\nu}\right)=B^{1 / 3} e^{-\nu}\left[\frac{1}{2}\left(\frac{V^{\prime \prime}}{V^{\prime}}-\frac{U^{\prime \prime}}{U^{\prime}}\right)+\frac{1}{2 B}\left(V^{\prime}-U^{\prime}\right)\left(\Lambda B^{4 / 3}-6 \mu\right)\right]
\end{aligned}
$$

Hence (22) tell us that given two arbitrary functions $U$ and $V$ we can determine $\mathrm{e}$ and т. We now show that only one of these functions is physical.

As a general rule any coordinate transformation in the bulk will shift our boundary making our wall evolving in time. However we can make a class of coordinate transformations in the bulk which leave our wall boundary conditions fixed, namely

$$
u \longrightarrow f(u), \quad v \longrightarrow f(v)
$$


i.e. the rescaling of the light-cone coordinates by some arbitrary function. This boundary invariance is a result of the two-dimensional conformal symmetry on the $t-z$ plane. Now we choose a particular coordinate transformation, $f \equiv V$, to fix this non-physical gauge freedom, thereby effectively setting $V \equiv v$. Hence there is only one remaining arbitrary function or physical degree of freedom, $U(u)$, which completely determines $\mathrm{e}$ and $\mathrm{T}$ for the wall.

There are now two equivalent ways, as it turns out, to look for cosmological solutions. We either, in the spirit of [12], consider a fixed boundary in the general spacetime (20), using the junction conditions (22), or we make coordinate transformations simplifying the bulk and study the moving wall trajectories in that case.

In fact, it turns out that the general class II solution (20) is a static spacetime. Setting

$$
r=B^{1 / 3} ; \quad t=3(v-U)
$$

(20) becomes,

$$
d s^{2}=h(r) d t^{2}-\frac{d r^{2}}{h(r)}-r^{2} d x_{I I I}^{2}
$$

where $h(r)$ has the familiar form

$$
h(r)=-\frac{B^{\prime} B^{-2 / 3}}{9}=\kappa-\frac{\Lambda}{6} r^{2}-\frac{\mu}{r^{2}}
$$

recognised as the generalised "Schwarzschild-AdS" solutions.

Note however that in making our background static our wall is no longer fixed, since the trajectory $\mathbf{z}=0$, or $u=v$, gives a nontrivial relationship between $r$ and $t: r=R(t)$ from (24). The reader should note here, that time dependence of the metric (20) in the bulk, portrayed by arbitrary function $U(u)$, and static boundary (22) has been transformed into time dependence for the boundary portrayed by the arbitrary trajectory $r=R(t)$, in a static bulk spacetime (25). Hence all essential information of the bulk metric is transferred onto the boundary conditions and vice-versa.

As a final remark, we note that the generalization to domain walls in arbitrary dimension is straightforward, simply altering the $\mu / r^{2}$ to $\mu / r^{(n-3)}$ in (26) (see [32] for a different approach).

\section{COSMOLOGICAL EVOLUTION EQUATIONS}

In this section we derive the equations of motion for a general (i.e. not necessarily symmetric) wall bounding two regions of spacetime with possibly differing cosmological constants. The reason for setting up the problem in its full generality is that we will be able to use the same set of equations to deal not only with non $Z_{2}$ symmetric walls, such as in the Millenium model, but also with walls which separate negative and zero cosmological constant spacetimes. This latter case will of course also require consideration of both class I and class II solutions for the $\Lambda=\kappa=0$ spacetime. We begin with a wall bounding two class II spacetimes. 
The starting point is the general solution (25). To get a wall solution, we simply take a general boundary

$$
X^{a}=(t(\tau), R(\tau), \chi, \theta, \phi)
$$

and compute the extrinsic curvatures on each side. Note that $\tau$ is the proper time with respect to an observer comoving with the wall, i.e.

$$
\dot{t}^{2} h(r)-\dot{R}^{2} h^{-1}(r)=1
$$

and is therefore well defined and intrinsic to the wall, whereas $t$ is the coordinate time in the bulk, and need not agree with the coordinate time on the other side of the wall. The only other subtlety arises in the choice of sign for the outward normal

$$
n_{a}= \pm(\dot{R}(\tau),-\dot{t}(\tau), \mathbf{0})
$$

This corresponds to the fact that we have a choice of which part of the spacetime we wish to keep (i.e. $r<R$ or $r>R$ ). This depends on the physical solution required, i.e. whether we have a positive or negative energy wall, and what sort of asymptotic solution we require. In general, positive energy walls match across two interior spacetimes and correspond to the lower (-) choice of sign in the outward normal and vice versa. It is also possible to have a wall matching an interior and exterior spacetime (for example the spherical collapsing wall, [22], used by Gogberashvili [33] in an early version of the RS scenario), however, for clarity in what follows unless explicitly stated, the formulae will be valid for $+(-)$ energy walls matching two interior (exterior) patches of the Schwarzschild-ADS spacetime.

Computing the extrinsic curvature components on each side of the wall gives

$$
\begin{aligned}
\mathrm{E} & =\mp \frac{3}{R}\left(\dot{t}_{+} h_{+}+\dot{t}_{-} h_{-}\right) \\
\frac{2}{3} \mathrm{E}-\mathrm{T} & = \pm\left(\frac{\ddot{R}+\frac{1}{2} h_{+}^{\prime}}{\dot{t}_{+} h_{+}}+\frac{\ddot{R}+\frac{1}{2} h_{-}^{\prime}}{\dot{t}_{-} h_{-}}\right)
\end{aligned}
$$

where $t_{ \pm}$represent the coordinate times on each side of the wall, $\dot{t}_{ \pm} h_{ \pm}$being given by (28) in each case. Note that we can also express (30b) in a different fashion as an energy conservation equation,

$$
\frac{D \mathrm{E}}{d \tau}+3 \frac{\dot{R}}{R}(\mathrm{E}-\mathrm{T})=0
$$

These represent the general equations of motion for an arbitrary wall separating two regions of spacetime with possibly different cosmological constants. Note that at this point $\mathrm{E}$ and $\mathrm{T}$ are unconstrained physically, and are simply given by the above expressions in terms of $R(\tau)$, which, other than representing a non-spacelike hypersurface, is completely arbitrary. This is worth stressing - that we can take any such $R(\tau)$, and we will get a wall solution with energy and tension determined by the embedding. Only when we impose physical constraints on our energy and tension do we get constraints on our possible hypersurface $R(\tau)$. Before exploring such constraints however, we simply note that (30) can also be expressed in terms of brane cosmological evolution equations (cf. Binetruy et. al. [12]) which are 


$$
\begin{aligned}
& \left(\frac{\dot{R}}{R}\right)^{2}=\frac{\mathrm{E}^{2}}{36}+\frac{9\left(\Delta k^{2}\right)^{2}}{4 \mathrm{E}^{2}}-\overline{k^{2}}-\frac{\kappa}{R^{2}}+\frac{\bar{\mu}}{R^{4}}-\frac{9 \Delta k^{2} \Delta \mu}{2 R^{4} \mathrm{E}^{2}}+\frac{9(\Delta \mu)^{2}}{4 \mathrm{E}^{2} R^{8}} \\
& \ddot{R}=-(2 \mathrm{E}-3 \mathrm{~T})\left(\frac{R \mathrm{E}}{36}+\frac{9 \Delta k^{2} \Delta \mu}{2 \mathrm{E}^{3} R^{3}}\right)+R\left(\frac{9\left(\Delta k^{2}\right)^{2}(4 \mathrm{E}-3 \mathrm{~T})}{4 \mathrm{E}^{3}}-\overline{k^{2}}\right)-\frac{\bar{\mu}}{R^{3}}-\frac{27 \mathrm{~T}(\Delta \mu)^{2}}{4 \mathrm{E}^{3} R^{7}}
\end{aligned}
$$

where we have now put $6 k_{ \pm}^{2}=-\Lambda_{ \pm}$. These are the completely general brane cosmological evolution equations for a brane separating two 'ADS' regions, and were first derived by Stoica et. al. [34].

Since we are particularly interested in the cosmological solutions for branes in models with regions of vacuum spacetime, we must also consider the case of a planar wall bordering on a class I spacetime which we may write as

$$
d s^{2}=H(v) d r d v-r^{2} d \mathbf{x}^{2}
$$

This spacetime is actually flat [30], as can be seen by the coordinate transformation

$$
\begin{aligned}
\mathbf{x}^{*} & =r \mathbf{x} \\
t^{*}-z^{*} & =r \\
t^{*}+z^{*} & =\int H d v+r \mathbf{x}^{2}
\end{aligned}
$$

which gives the Minkowski metric in the starred coordinate system.

Clearly to match with an ADS spacetime we need to set $r=R(\tau)$, and compute the relevant extrinsic curvature quantities. For a wall bounding a class II and class I spacetime, we find that the junction conditions are now

$$
\begin{aligned}
\mathrm{E} & =\mp \frac{3}{R}\left(\dot{R}+\dot{t}_{-} h_{-}\right) \\
\frac{2}{3} \mathrm{E}-\mathrm{T} & = \pm\left(\frac{\ddot{R}}{\dot{R}}+\frac{\ddot{R}+\frac{1}{2} h_{-}^{\prime}}{\dot{t}_{-} h_{-}}\right)
\end{aligned}
$$

Therefore we now find a somewhat different set of cosmological equations for the evolution of the wall:

$$
\begin{aligned}
\frac{\dot{R}}{R} & = \pm\left(\frac{3 k^{2}}{2 \mathrm{E}}-\frac{\mathrm{E}}{6}-\frac{3 \mu_{-}}{2 \mathrm{E} R^{4}}\right) \\
\ddot{R} & =\frac{k^{2} R}{2 \mathrm{E}^{2}}\left(9 k^{2}-\mathrm{E}^{2}\right)+\frac{(2 \mathrm{E}-3 \mathrm{~T}) R}{36 \mathrm{E}^{3}}\left(81 k^{4}-\mathrm{E}^{4}\right)+\frac{\mu_{-}}{2 \mathrm{E}^{3} R^{3}}\left[9 k^{2}(3 \mathrm{~T}-2 \mathrm{E})-\mathrm{E}^{3}\right]-\frac{27 \mathrm{~T} \mu_{-}^{2}}{4 \mathrm{E}^{3} R^{7}}
\end{aligned}
$$

where we see that the 'Friedman' equation is now linear in $\dot{R}$.

Finally, if we are matching two class I spacetimes, the junction conditions give the particularly simple relations

$$
\begin{aligned}
& \mathrm{E}=\mp \frac{6 \dot{R}}{R} \\
& \mathrm{~T}=\mp\left(\frac{4 \dot{R}}{R}+\frac{2 \ddot{R}}{\dot{R}}\right)
\end{aligned}
$$


In the next section we will compute cosmological trajectories for general walls, however, before doing so it is useful to verify that this approach is indeed valid by cross-checking it against a few simple known solutions. For example, a planar symmetric domain wall in pure Einstein gravity is known to have a particularly simple interpretation in terms of the matched interiors of two hyperboloids in Minkowski spacetime [35]. In the context of these equations, a $\Lambda=\kappa=0$ spacetime can match class I or class II spacetimes. For the matching of two class I spacetimes we must use (37) with $\mathrm{E}=$ т. Clearly this has the solution $R=e^{\mathrm{E} \tau / 6}$, giving a de-Sitter like induced metric in agreement with the standard domain wall metric in wall-based coordinates (see e.g. [22,36]). Inverting this relation to find $R(v)$ in terms of the bulk coordinates gives $R=-18 / \mathrm{E}^{2} v$. Finally, inverting (34) gives the particularly simple bulk equation of motion for the domain wall trajectory: $t^{* 2}-z^{* 2}-\mathbf{x}^{* 2}=-36 / \mathrm{E}^{2}$, i.e. a hyperboloid in Minkowski spacetime. The 'planar' domain wall is therefore a boundary between the interior of two Minkowski hyperboloids as required.

\section{COSMOLOGICAL WALL SOLUTIONS}

In this section we apply the general equations worked out in the previous section to find a variety of cosmological solutions. Let us begin by considering the particularly simple equation of state of a domain wall, $\mathrm{E}=\mathrm{T}$, and find the most general trajectories. First of all, note that (31) implies that $\mathrm{E}$ is a constant, therefore the brane-Friedmann equation, (32a), can be written as

$$
\frac{R^{4}}{4}\left(\frac{d R^{2}}{d \tau}\right)^{2}=a R^{8}-\kappa R^{6}+b R^{4}+c
$$

where

$$
\begin{aligned}
a & =\frac{\mathrm{E}^{2}}{36}+\frac{9\left(\Delta k^{2}\right)^{2}}{4 \mathrm{E}^{2}}-\overline{k^{2}} \\
b & =\bar{\mu}-\frac{9 \Delta k^{2} \Delta \mu}{2 \mathrm{E}^{2}} \\
c & =\frac{9(\Delta \mu)^{2}}{4 \mathrm{E}^{2}}
\end{aligned}
$$

which can be integrated in general, giving $R^{2}$ in terms of elliptic functions, although the expression is not particularly illuminating. If one of $\kappa, c$ is zero, the solutions are very simple to write down, but for general $a, b, c, \kappa$, the qualitative behaviour of the solutions can be deduced from (38). The constant ' $a$ ' can be seen to measure the departure from criticality of the domain wall - i.e. that value which allows a static planar domain wall solution in the absence of an ADM mass term. We will therefore denote $a=0$ walls as critical, and $a>(<) 0$ walls as super-(sub-)critical.

Clearly, if there is a nonzero ADM mass in the bulk there is always a solution which expands outward from an initial singularity, $R=0$, which is where the wall trajectory touches the central singularity of the black hole. Whether or not this solution expands indefinitely, or there is a final singularity depends on the roots of (38). For $a \geq 0$, there are zero or two (possibly repeated) roots for $R^{2}>0$. If $b>\frac{9}{32 a}$ for $\kappa=1$, or $b>0$ otherwise, 
then there are no positive roots and the cosmology expands indefinitely. Otherwise there can be two separate roots, in which case there is a cosmology with initial and final singularities, and a nonsingular cosmology which contracts in to a minimum radius and re-expands. If there is a repeated root, $R_{c}^{2}$, then the cosmology asymptotes $R_{c}$ exponentially at late times either as a contracting branch, or an expanding branch with an initial singularity. If $a<0$ there is always one root and our universe starts and ends its life on the black hole singularity. Finally, there is always a static solution at any positive root of the quartic.

For planar domain walls, i.e. $\kappa=0$, we have the exact solution

$$
R^{4}= \begin{cases}\frac{1}{4 a}\left(e^{4 \sqrt{a}\left(\tau-\tau_{0}\right)}-2 b+\left(b^{2}-4 a c\right) e^{-4 \sqrt{a}\left(\tau-\tau_{0}\right)}\right) & a>0 \\ \frac{\sqrt{b^{2}-4 a c}}{2|a|} \sin \left\{4 \sqrt{|a|}\left(\tau-\tau_{0}\right)\right\}-\frac{b}{2|a|} & a<0 \\ 4 b\left(\tau-\tau_{0}\right)^{2}-\frac{c}{b} & a=0\end{cases}
$$

which clearly demonstrates the expansion outwards from an initial singularity, and for $a>0$ shows the late time inflationary nature of the cosmology.

If $c=0$, i.e. the mass terms on either side of the wall are the same, the exact solutions are

$$
R^{2}= \begin{cases}\frac{1}{4 a}\left(e^{2 \sqrt{a}\left(\tau-\tau_{0}\right)}+2 \kappa+\left(\kappa^{2}-4 a b\right) e^{-2 \sqrt{a}\left(\tau-\tau_{0}\right)}\right) & a>0 \\ \frac{\sqrt{\kappa^{2}-4 a b}}{2 a} \sin \left\{2 \sqrt{|a|}\left(\tau-\tau_{0}\right)\right\}+\frac{\kappa}{2 a} & a<0 \\ -\kappa\left(\tau-\tau_{0}\right)^{2}+\frac{b}{\kappa} & a=0\end{cases}
$$

where we have taken $\kappa \neq 0$ : if $\kappa=0$ use (40). A nice analysis of supercritical domain wall solutions and their bulk embeddings was given by Khoury et. al. in [37].

For the case of a general wall we need some sort of equation of state $\mathrm{T}(\mathrm{E})$, for which we will follow convention in setting

$$
\mathrm{E}=\mathrm{E}_{0}+\rho \quad ; \quad \mathrm{T}=\mathrm{E}_{0}-p
$$

where $p=(\gamma-1) \rho$ would be a typical equation of state, although we re-emphasize that for $\rho \simeq \mathrm{E}$ there is no reason to suppose that such an equation of state will be accurate, or even possible. The conservation of energy momentum (31) implies that $\rho=\rho_{0} R^{-3 \gamma}$ and hence $\mathrm{E}$ is no longer a constant. In order to deal with this additional difficulty (in the non- $Z_{2}$-symmetric case) we keep only terms linear in $\rho$ which boils down to looking at wall trajectories for late times. For example in the case of radiation cosmology, $\gamma=4 / 3$, the Friedmann equation (32a) reduces to,

$$
\left(\frac{\dot{R}}{R}\right)^{2}=\frac{\mathrm{E}_{0}^{2}}{36}+\frac{9\left(\Delta k^{2}\right)^{2}}{4 \mathrm{E}_{0}^{2}}-\overline{k^{2}}-\frac{\kappa}{R^{2}}+\frac{1}{R^{4}}\left(\bar{\mu}-\frac{9 \Delta k^{2} \Delta \mu}{2 \mathrm{E}_{0}^{2}}+\frac{\mathrm{E}_{0} \rho_{0}}{18}-\frac{9 \rho_{0}\left(\Delta k^{2}\right)^{2}}{2 \mathrm{E}_{0}^{3}}\right)+O\left(R^{-8}\right)
$$

The above equation has exactly the same solution as (41) for $\kappa \neq 0$ where however the constants $a$ and $b$ are given by,

$$
a=\frac{\mathrm{E}_{0}^{2}}{36}+\frac{9\left(\Delta k^{2}\right)^{2}}{4 \mathrm{E}_{0}^{2}}-\overline{k^{2}} \quad ; \quad b=\bar{\mu}-\frac{9 \Delta k^{2} \Delta \mu}{2 \mathrm{E}_{0}^{2}}+\frac{\mathrm{E}_{0} \rho_{0}}{18}-\frac{9 \rho_{0}\left(\Delta k^{2}\right)^{2}}{2 \mathrm{E}_{0}^{3}} \quad ;
$$


Another important class of solutions are those with $Z_{2}$ symmetry, for which the cosmological Friedman equation has the form of (38) with the differences ' $\Delta$ ' of all quantities being zero. The Friedmann equation (32a) becomes

$$
\left(\frac{\dot{R}}{R}\right)^{2}=\frac{\mathrm{E}_{0}^{2}}{36}-k^{2}-\frac{\kappa}{R^{2}}+\frac{\mathrm{E}_{0} \rho_{0}}{18 R^{3 \gamma}}+\frac{\mu}{R^{4}}+\frac{\rho_{0}^{2}}{36 R^{6 \gamma}}
$$

where the above is now an exact expression. For example, a radiation cosmology would have $\gamma=4 / 3$, and the equation of motion would be as (38), but with the constants $a_{r}, b_{r}, c_{r}$, now defined as

$$
a_{r}=\frac{\mathrm{E}_{0}^{2}}{36}-k^{2} \quad ; \quad b_{r}=\mu+\frac{\mathrm{E}_{0} \rho_{0}}{18} \quad ; \quad c_{r}=\frac{\rho_{0}^{2}}{36}
$$

and so a generic planar solution would be given by (40) with these new values for the constants $a, b$, and $c$. We see now the interplay between the contribution of the cosmological matter energy density $\rho_{0}$, which contributes to both $b$ and $c$, and the "CFT" contribution, $\mu$, which appears only in $b$. This changes the way in which the initial singularity, $R=0$, is approached, for example, Gubser's solution has $a=0=\rho_{0}$, and $R=\mu^{\frac{1}{4}} \sqrt{2\left(\tau-\tau_{0}\right)}$, as opposed to $R=b^{\frac{1}{4}}\left[4\left(\tau-\tau_{0}\right)^{2}-c / b^{2}\right]^{\frac{1}{4}}$. A description of $Z_{2}$ symmetric cosmological solutions and their embeddings was given by Mukohyama et. al. [17].

Let us now turn to the less well-explored question of cosmological solutions in models with slices of vacuum and ADS spacetimes, such as the noncompact GRS model, or compact models such as those in 28,38. Without loss of generality, we will take $k_{+}=0, k_{-}=k$, for which the general equations of motion are given by (32) or (36). For simplicity, since we are unlikely to be interested in cosmologies on negative tension walls, let us consider the case where the boundary has the equation of state of a domain wall, i.e. $\mathrm{E}=\mathrm{T}$, and let $\varepsilon=-(\mathrm{E}+3 k)$ be the departure from 'criticality' of this domain wall $(\varepsilon>0$ being supercritical).

We start by considering the matching of two class II spacetimes. Note that for $\kappa=$ $0,-1$, in order to have the correct spacetime signature, we require $\mu_{+}<0$, and we have a naked singularity in the full bulk solution. For $\kappa=1, \mu_{+}>0$ gives the five-dimensional Schwarzschild solution.

Substituting the values of $k_{ \pm}$gives for the constants in (38)

$$
a=\frac{\varepsilon^{2}}{36 \mathrm{E}^{2}}(\varepsilon+2 \mathrm{E})^{2} ; b=\mu_{+}+\frac{\varepsilon(\varepsilon+2 \mathrm{E}) \Delta \mu}{2 \mathrm{E}^{2}} ; c=\frac{9(\Delta \mu)^{2}}{4 \mathrm{E}^{2}}
$$

Note that unlike the generic domain wall case, $a>0$ for both sub and super-critical walls.

For $\kappa=1, \mu_{+}>0$ and we again have solutions which expand out (or contract into) the singularity at $r=0$. Depending on the roots of the quartic, there are solutions which expand indefinitely, recontract, or indeed asymptote a constant radius. The critical domain wall always recollapses. For $\kappa=-1, \mu_{+}<0$, and the qualitative behaviour is similar, however the critical domain wall now expands indefinitely if $27 c>-4 \mu_{+}$.

For the planar $(\kappa=0)$ walls, the exact solutions are

$$
R^{4}= \begin{cases}\frac{1}{4 a}\left(e^{ \pm 4 \sqrt{a}\left(\tau-\tau_{0}\right)}-2 b+\left(b^{2}-4 a c\right) e^{\mp 4 \sqrt{a}\left(\tau-\tau_{0}\right)}\right) & a>0 \\ 4 b\left(\tau-\tau_{0}\right)^{2}-\frac{c}{b} & a=0\end{cases}
$$



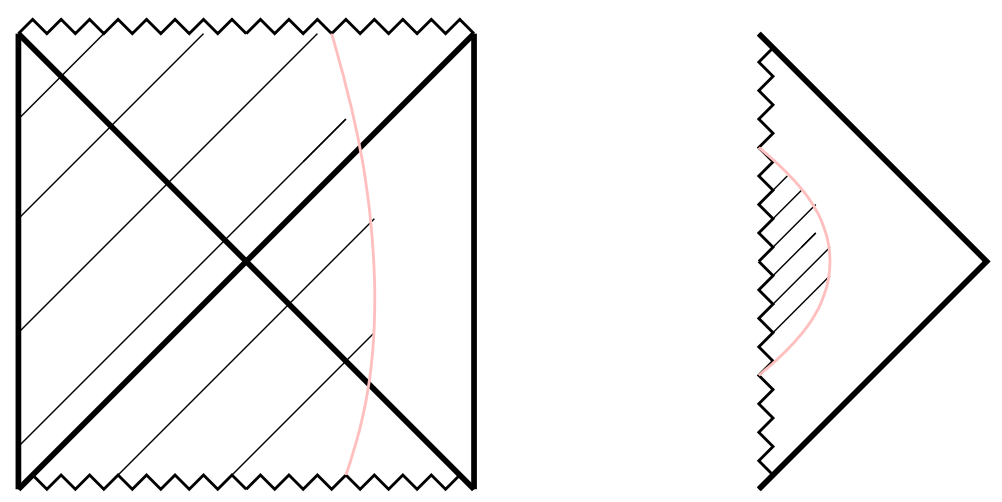

FIG. 1. A negative tension domain wall patching vacuum to Schwarzschild-ADS spacetime. The vacuum bulk has a naked singularity.

For the critical domain wall, $b=\mu_{+}<0$, and we see that the domain wall universe has an initial and final singularity, for $\tau_{0}-\frac{\Delta \mu}{4 k \mu_{+}}<\tau<\tau_{0}+\frac{\Delta \mu}{4 k \mu_{+}}$. Inverting (28) to find the trajectory in terms of coordinate time in the vacuum spacetime to the right of the wall shows that these singularities occur at finite coordinate time, and the wall actually expands out of, and re-collapses into, the central naked singularity in this spacetime. This corresponds to figure 1, and would appear to be a rather undesirable spacetime from the five-dimensional point of view.

For the subcritical domain wall however, $b<0$ with $b^{2}-4 a c>0$, hence the cosmology has no initial singularity, but merely follows a modestly corrected sinh trajectory. For the supercritical domain wall, there are two possibilities, depending on the relative magnitudes of $\mu_{ \pm}$. If $9 k^{2}\left|\mu_{+}\right| \geq \mu_{-}\left(\varepsilon^{2}+6 k \varepsilon\right)$, then the cosmology is completely nonsingular as for the subcritical case, however, if $9 k^{2}\left|\mu_{+}\right|<\mu_{-}\left(\varepsilon^{2}+6 k \varepsilon\right)$, then there is an initial (or final) singularity with the universe inflating away (deflating towards) it.

Finally, for a planar asymptotically vacuum spacetime, the other possibility is matching to a class I spacetime for which we need (36). Setting $\mathrm{E}=\mathrm{T}=-\varepsilon-3 k<0$ again we have

$$
\frac{\dot{R}}{R}=\frac{\varepsilon(\varepsilon+2 \mathrm{E})}{6 \mathrm{E}}-\frac{3 \mu_{-}}{2 \mathrm{E} R^{4}}
$$

which has the general solution

$$
R^{4}= \begin{cases}\exp \left\{\frac{2 \varepsilon(\varepsilon+2 \mathrm{E})}{3 \mathrm{E}}\left(\tau-\tau_{0}\right)\right\}+\frac{9 \mu_{-}}{\varepsilon(\varepsilon+2 \mathrm{E})} & \varepsilon \neq 0 \\ \frac{2 \mu_{-}}{k}\left(\tau-\tau_{0}\right) & \varepsilon=0\end{cases}
$$

A supercritical wall therefore has $\dot{R}>0$ and expands outward from an initial singularity (where it touches the Schwarzschild singularity on the $(-)$ side of the wall). A subcritical wall however has $\dot{R}_{<}^{>0}$ for $R_{>}^{4<} \frac{9 \mu_{-}}{\varepsilon(\varepsilon+2 \mathrm{E})}$. This shows that there are two solutions, one of which has an inital singularity and expands outward to $R_{c}^{4}=\frac{9 \mu_{-}}{\varepsilon(\varepsilon+2 \mathrm{E})}$, and another which contracts to $R_{c}$. A critical domain wall has a power law expansion outward from an initial singularity.

It is interesting to transform into the starred coordinate system to follow these wall trajectories in Minkowski spacetime: 

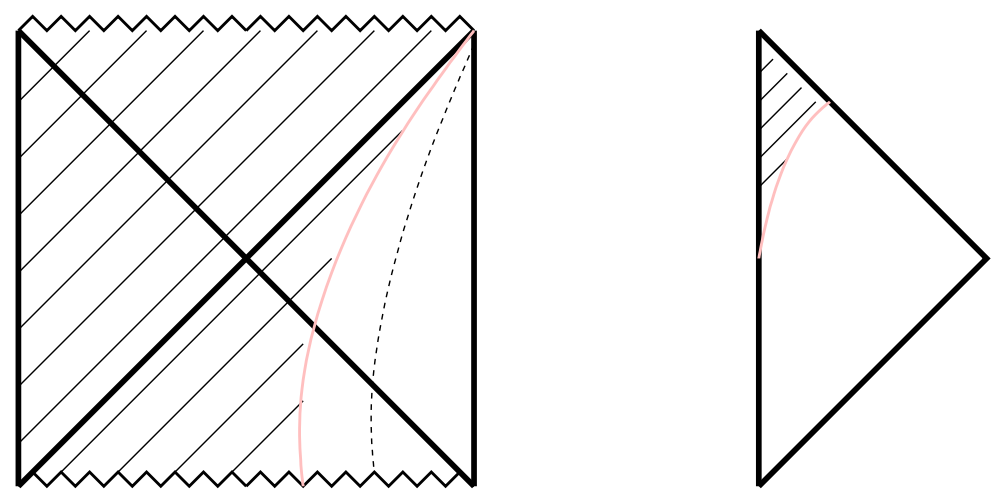

FIG. 2. A negative tension domain wall patching a class I (flat) vacuum spacetime to Schwarzschild-ADS. The dotted line indicates a possible additional positive energy cosmological domain wall.

$$
\begin{aligned}
\mathbf{x}^{*} & =R(\tau) \mathbf{x} \\
t^{*}-z^{*} & =R(\tau) \\
t^{*}+z^{*} & =\int \frac{d \tau}{\dot{R}(\tau)}+R(\tau) \mathbf{x}^{2}=F(R(\tau))+R(\tau) \mathbf{x}^{2}
\end{aligned}
$$

For a critical domain wall $F(R)=4 k^{2} R^{7} / 7 \mu_{-}^{2}$, and for a noncritical domain wall bordering pure ADS, $F(R)=-1 / 4 R$. We see therefore that this latter case is the familiar hyperboloid in Minkowski spacetime, although it is now the interior which is excised for this negative tension wall.

The critical domain wall satisfies

$$
\mathbf{x}^{* 2}=t^{* 2}-z^{* 2}-\frac{4 k^{2}}{7 \mu_{-}^{2}}\left(t^{*}-z^{*}\right)^{8}
$$

which is a deformed hyperboloid. At fixed time, the 3-sphere is now squashed into an eggshape in the $\left|\mathbf{x}^{*}\right|, z^{*}$ directions. Again, it is the interior of this squashed $\mathrm{S}^{3}$ which is excised. On the ADS side, the wall expands outward from the initial black hole singularity and at late times expands as $R \propto t_{-}^{1 / 3}$ (c.f. $R \propto t$ for Gubser's critical $Z_{2}$ symmetric solution). The appropriate matching is shown in figure 2 .

Finally it is worth stressing that these trajectories are independent of whether or not there is an additional positive tension wall present - the existence of the wall trajectory is a local question. We can therefore add in a positive domain wall in the usual way by simply adding another boundary to the Schwarzschild ADS spacetime as shown by the dotted line in figure 2 .

\section{DISCUSSION}

Starting from the most general five-dimensional metric with homogeneous and isotropic spatial 3-sections, we have shown that the most general cosmological brane solution matches two Schwarzschild-ADS spacetimes, or a Schwarzschild-ADS spacetime with a class I or II vacuum spacetime. It is worth emphasizing that the three dimensional homogeneity and 
isotropy ensures that the bulk metric solution is invariant under two dimensional conformal symmetries. By inputting a boundary or wall we in principle break these conformal symmetries, however, it turns out that the junction conditions are such that only half are broken. This in turn ensures that the only remaining degree of freedom is the wall's trajectory itself. Hence all the essential information of the wall's dynamics is contained on the wall trajectory itself (the boundary) the bulk being a specific static spacetime playing a 'background' role.

We then derived the generalised FRW equations for a wall with general energy and tension, then applied these to a range of cases, focusing on the previously unexplored case of a wall bordering a vacuum region on one side. The interesting feature of these latter solutions is that if two class II spacetimes are matched, the bulk can contain timelike naked singularities on the vacuum side of the wall. For a more satisfactory nonsingular solution, one must match the planar wall to a class I spacetime. Here the wall trajectories are reminiscent of the vacuum domain wall of Vilenkin, Ipser and Sikivie, in that they are deformed hyperboloids, although it is the interior of the hyperboloid which is excised for this negative energy wall.

If we now wish to construct a 'cosmological' GRS solution, i.e. one which has a $Z_{2}$ symmetric positive energy central wall with matter residing on it, and a negative energy outer wall, then we must combine one of the $Z_{2}$ solutions of (45) with a class II/II or class II/I solution for the outer wall. In each case, we must be careful to keep the (-) wall to the right of the $(+)$ wall, $R_{-}<R_{+}$. For example, if we take the planar domain wall, the central domain wall trajectory, $R_{+}$, is given by (40) with the constants $a, b, c$ in (46). Using the class II/I solutions of (48) for the (-) wall shows that we cannot match arbitrary walls, since for example a subcritical central wall would collide with a critical or supercritical outer wall bringing our universe to an abrupt and catastrophic end. Two generic critical walls are compatible however, with

$$
\left\{\begin{array}{l}
R_{+}^{4}=4\left(\mu+\frac{k \rho_{0}}{3}\right)\left(\tau_{+}-\tau_{0+}\right)^{2}-\frac{\rho_{0}^{2}}{36 \mu+12 k \rho_{0}} \\
R_{-}^{4}=\frac{2 \mu}{k}\left(\tau_{-}-\tau_{0^{-}}\right)
\end{array}\right.
$$

as is a critical central wall with a subcritical outer one. A supercritical central wall can exist with a critical or subcritical outer wall, or a supercritical outer wall with $2 \sqrt{E_{+}^{2}-36 k^{2}}>$ $\left(9 k^{2}-\mathrm{E}_{-}^{2}\right) / \mathrm{E}_{-} \mid$; and a subcritical central wall can exist with a subcritical outer wall provided $\frac{\sqrt{b_{r}^{2}-4 a_{r} c_{r}}-b_{r}}{2\left|a_{r}\right|}>\frac{9 \mu}{\left(9 k^{2}-\mathrm{E}_{-}^{2}\right)}$.

For example we can match the critical Gubser solution, $R_{+}^{4}=4 \mu \tau_{+}^{2}$, with the critical class II/I solution $R_{-}^{4}=2 \mu \tau_{-} / k$. However, a pure critical planar radiation cosmology, $R_{+}^{4}=\rho_{0}\left(16 k^{2} \tau_{+}^{2}-1\right) / 12 k$ cannot be matched to any class I asymptotically flat spacetime across a (-) wall, as the only possible solution is an exponentially expanding or contracting one for a super/sub critical wall respectively.

Therefore, while there are some constraints on the central wall cosmologies, these are not overwhelmingly restrictive.

This leads us to the issue of the radion in these cosmological models. There has been a great deal of debate about the radion in the context of cosmological models (see e.g. [29]) and in the case of quasi-localised gravity (see e.g. [25 27]). We have not supposed any stabilization mechanism, [39], for the extra dimension, but simply looked for free cosmological 
solutions. Since the radion leads to anti-gravity in the original GRS model, it is interesting to see how it manifests itself here.

The wavefunction of the radion is straightforward to find in linearized gravity, [40, and it behaves as a scalar field living on the brane worldvolume which satisfies a massless equation of motion, $\partial^{2} f=0$. In the context of a homogeneous and isotropic cosmology, this has the simple solution $f=f_{0} t$, where $t$ is the coordinate time on the brane, and we have chosen to have zero displacement for $t=0$. Now consider a supercritical $Z_{2}$-symmetric positive energy planar domain wall in pure ADS spacetime; this follows a trajectory

$$
R=\exp \left\{\sqrt{\mathrm{E}^{2}-36 k^{2}}\left(\tau-\tau_{0}\right) / 6\right\}=\left[1-\frac{k^{2} \sqrt{\mathrm{E}^{2}-36 k^{2}}}{\mathrm{E}}\left(t-t_{0}\right)\right]^{-1}
$$

in terms of either the brane proper time, $\tau$, or the local bulk coordinate time, $t$. We see therefore that for small $t$ and $\sqrt{\mathrm{E}^{2}-36 k^{2}}$,

$$
R \simeq 1+\frac{k \sqrt{3 k} \sqrt{\mathrm{E}-6 k}}{3}\left(t-t_{0}\right)
$$

we can therefore identify $f_{0}=k^{3 / 2} \sqrt{\mathrm{E}-6 k} \sqrt{3}$, i.e. $\delta_{\mathrm{E}}=\mathrm{E}-6 k=3 f_{0}^{2} k^{-3 / 2}$, which has the correct dependence on $f_{0}$ for a standard energy momentum tensor for the radion. (Note we cannot have a subcritical planar domain wall.) This means that a negative energy wall requires $\mathrm{E}<-6 k$, and nominally a negative radion energy on that wall.

Obviously these solutions do not see any evidence of the fifth dimension "opening up" [24], or any instability due to the radion, however, this is because of the high degree of symmetry of the set-up. If we were to break isotropy or homogeneity, the integrable nature of the system would likely be destroyed, and the simplicity of the description of the wall as a trajectory in bulk Schwarzschild-ADS would disappear. Destruction of these symmetries would lead to a brane-bulk interaction (as evidenced by the extreme case of the as yet undiscovered black hole on the brane solution - see [41] for discussions on this topic) through which the more familiar effects of the radion would be recovered.

Of course it is tempting to enquire whether the so-called "missing mode" of free motion of a wall can be identified in this set-up. For a non-gravitating defect, the effective equation of motion is given by the relation $\bar{K}=0$, i.e. the wall (in this case) is a minimal hypersurface. An equivalent perturbative description would state that the displacement of the wall satisfies a massless wave equation on the wall. There have been claims 42 in the literature that when gravity is included this 'free motion' disappears, as would appear to agree with the rejection of the free 'radion' solution 40]

$$
\delta g_{\mu \nu}=\frac{\left(r^{-2}-r^{2}\right)}{4 k} \bar{K}_{\mu \nu}
$$

in the usual Randall-Sundrum perturbation analysis. However, in this simple picture, each wall has its own 'radion' corresponding to motion through the ambient spacetime. Indeed, a perturbative analysis of the thick four-dimensional domain wall 43 indicates no such disappearance of this motion with the coupling to gravity. What therefore can be going on? A clue perhaps lies in the effective equation of state for a freely moving wall. As Carter and Vilenkin [44, argued for the cosmic string, a freely moving defect will have an 
altered effective equation of state. The effective energy per unit area will increase, and the tension will decrease. Generalizing the Carter-Vilenkin formula for the domain wall gives the effective equation of state

$$
\mathrm{ET}^{3}=\mathrm{E}_{0}^{4}
$$

for a 3-brane domain wall. Clearly for $\mathrm{E}$ close to $\mathrm{E}_{0}$, this gives an equation of state of a radiation cosmology on the background domain wall. Now we see how a freely moving wall might no longer be a small perturbation of a 'straight' one: the averaged equation of state of the freely moving wall causes the wall to follow a centre of mass trajectory associated with a radiation universe, which is not, at late times (when the radiation universe approximation is particularly good) a small perturbation of the unmoving wall. Of course, a freely moving wall will not be isotropic and homogeneous except at the very large scale, and it is also possible that there are brane-bulk interactions which further complicate the issue.

Finally, the problem of brane cosmological perturbation theory is very important to understand if we wish to do real cosmology. The setting up of the formalism for perturbation theory on the brane is already underway 44,46]; by considering the cosmological domain wall in terms of its global spacetime structure, the role of the bulk, its interactions with matter on the brane, and the interpretation of some of the gauge invariant variables in terms of bulk physical quantities will be more clearly elucidated.

\section{ACKNOWLEDGEMENTS}

It is a great pleasure to thank Roberto Emparan, David Fairlie, Jihad Mourad, Simon Ross, Valery Rubakov, and Douglas Smith for helpful and enlightening discussions.

C.C. was supported by PPARC, and R.G. by the Royal Society. 


\section{REFERENCES}

[1] L.Randall and R.Sundrum, Phys. Rev. Lett. 833370 (1999). hep-ph/9905221

L.Randall and R.Sundrum, Phys. Rev. Lett. 834690 (1999). hep-th/9906064

[2] V.A.Rubakov and M.E.Shaposhnikov, Phys. Lett. 125B 136 (1983).

K.Akama, in Gauge Theory and Gravitation. Proceedings of the International Symposium, Nara, Japan, 1982, eds. K.Kikkawa, N.Nakanishi and H.Nariai (Springer-Verlag, 1983).

[3] M.Visser, Phys. Lett. 159B 22 (1985). hep-th/9910093.

E.J.Squires, Phys. Lett. 167B 286 (1986).

[4] A.G.Cohen and D.B.Kaplan, Phys. Lett. 470B 52 (1999). hep-th/9910132

R.Gregory, Phys. Rev. Lett. 842564 (2000). hep-th/9911015

T.Gherghetta and M.Shaposhnikov, Phys. Rev. Lett. 85240 (2000). [hep-th/0004014

M.Gogberashvili and P.Midodashvili, Brane universe in six dimensions, hepph/0005298.

I.Olasagasti and A.Vilenkin, Gravity of higher-dimensional global defects, hepth/0003300.

[5] P.Horava and E.Witten, Nucl. Phys. B475 94 (1996). hep-th/9603142

[6] A.Lukas, B.A.Ovrut, K.S.Stelle and D.Waldram, Phys. Rev. D59 086001 (1999). hepth/9803235

[7] A.Lukas, B.A.Ovrut and D.Waldram, Phys. Rev. D60 086001 (1999). hep-th/9806022

[8] H.Reall, Phys. Rev. D59 103506 (1999). hep-th/9809195

[9] J.Garriga and T.Tanaka, Phys. Rev. Lett. 842778 (2000). hep-th/9911055

[10] S.Giddings, E.Katz and L.Randall, JHEP 0003:023 (2000). [hep-th/0002091]

[11] P.Binetruy, C.Deffayet and D.Langlois, Nucl. Phys. B565 269 (2000). hep-th/9905012

[12] P.Binetruy, C.Deffayet, U.Ellwanger and D.Langlois, Phys. Lett. 477B 285 (2000). [hepth/9910219

[13] N.Kaloper, Phys. Rev. D60 123506 (1999). hep-th/9905210

D.J.Chung and K.Freese, Phys. Rev. D61 023511 (2000). [hep-ph/9906542

C.Csaki, M.Graesser, C.Kolda and J.Terning, Phys. Lett. 462B 34 (1999). hep ph/9906513

J.Cline, C.Grojean and G.Servant, Phys. Rev. Lett. 834245 (1999). hep-ph/9906523

[14] T.Shiromizu, K.-I.Maeda and M.Sasaki, The Einstein equations on the 3-brane world, gr-qc/9910076.

[15] R.Maartens, Cosmological dynamics on the brane, hep-th/0004166.

[16] D.Ida, Brane world cosmology, gr-qc/9912002.

[17] S.Mukohyama, T.Shiromizu and K.-I.Maeda, Phys. Rev. D62 024028 (2000). hepth/9912287

[18] P.Kraus, JHEP 9912:011 (1999). hep-th/9910149

[19] H.A.Chamblin and H.Reall, Nucl. Phys. B562 133 (1999). hep-th/9903225]

H.A.Chamblin, M.J.Perry and H.Reall, JHEP 9909:014 (1999). hep-th/9908047

[20] S.Gubser, ADS/CFT and gravity, hep-th/9912001.

S.W.Hawking, T.Hertog and H.S.Reall, Phys. Rev. D62 043501 (2000). hepth/0003052

L.Anchordoqui, C.Nunez and K.Olsen, Quantum cosmology and AdS/CFT, hepth/0007064. 
[21] J.Lykken and L.Randall, JHEP 0006:014 (2000). [hep-th/9908076]

[22] J. Ipser and P. Sikivie, Phys. Rev. D30 712 (1984).

[23] I.I.Kogan, S.Mouslopoulos, A.Papazoglou and G.G.Ross, A three three-brane universe: new phenomenology for the new millenium?, hep-ph/9912552.

[24] R.Gregory, V.A.Rubakov and S.Sibiryakov, Phys. Rev. Lett. 84 5928-5931 (2000). hepth/0002072

[25] C.Csaki, J.Erlich and T.J.Hollowood, Phys. Rev. Lett. 845932 (2000). hep-th/0002161 G.Dvali, G.Gabadadze and M.Porrati, Phys. Lett. 484B 112 (2000). hep-th/0002190

C.Csaki, J.Erlich and T.J.Hollowood, Phys. Lett. 481B 107 (2000). hep-th/0003020

[26] R.Gregory, V.A.Rubakov and S.Sibiryakov, Gravity and antigravity in a brane world with metastable gravitons, hep-th/0003045.

L.Pilo, R.Rattazzi and A.Zaffaroni, The fate of the radion in models with metastable graviton, hep-th/0004028.

[27] C.Csaki, J.Erlich, T.J.Hollowood and J.Terning, Holographic RG and cosmology in theories with quasilocalized gravity, hep-th/0003076.

[28] I.I.Kogan, S.Mouslopoulos, A.Papazoglou and G.G.Ross, Multi-brane worlds and modification of gravity at large scales, hep-th/0006030.

[29] P.Kanti, I.I.Kogan, K.A.Olive and M.Pospelov, Phys. Lett. 468B 31 (1999). hhepph/9909481|

E.Flanagan, S.H.Tye and I.Wasserman, A cosmology of the brane world, hepph/9909373.

E.Flanagan, S.H.Tye and I.Wasserman, Cosmological expansion in the Randall-Sundrum brane world scenario, hep-ph/9910498.

C.Csaki, M.Graesser, L.Randall and J.Terning, Cosmology of brane models with radion stabilization, hep-ph/9911406.

H.Collins and B.Holdom, Brane cosmologies without orbifolds, hep-ph/0003173.

C.Barcelo and M.Visser, Phys. Lett. 482B 183 (2000). hep-th/0004056

[30] A.H.Taub, Ann. Math. 53472 (1951).

[31] W.Israel, Nuovo Cim. 44B 1 (1966).

[32] M.Cvetic and J.Wang, Phys. Rev. D61 124020 ((2000)). [hep-th/9912187]

[33] M.Gogberashvili, Europhys. Lett. 49396 (2000). hep-ph/9812365

[34] H.Stoica, S.H.Tye and I.Wasserman, Phys. Lett. 482B 205 (2000). hep-th/0004126

[35] G.W.Gibbons, Nucl. Phys. B394 3 (1993).

M.Cvetic, S.Griffies and H.Soleng, Phys. Rev. Lett. 71670 (1993). [hep-th/9212020

M.Cvetic, S.Griffies and H.Soleng, Phys. Rev. D48 2613 (1993). gr-qc/9306005

[36] A.Vilenkin, Phys. Lett. 133B 177 (1983).

[37] J.Khoury, P.Steinhardt and D.Waldram, Inflationary solutions in the brane world and their geometrical interpretation, hep-th/0006069.

[38] J.Lykken, R.C.Myers and J.Wang, Gravity in a box, hep-th/0006191.

[39] W.Goldberger and M.Wise, Phys. Rev. Lett. 834922 (1999). [hep-ph/9907447]

D.Choudhury, D.P.Jatkar, U.Mahanta and S.Sur, On the stability of the three 3-brane model, hep-ph/0004233.

J.E.Kim and B.Kyae, hep-th/0005139.

[40] C.Charmousis, R.Gregory and V.A.Rubakov, Wave function of the radion in a brane world, hep-th/9912160. 
[41] A.Chamblin, S.W.Hawking and H.S.Reall, Phys. Rev. D61 065007 (2000). hepth/9909205

R.Emparan, G.T.Horowitz and R.C.Myers, JHEP 0001:007 (2000). hep-th/9911043

R.Emparan, G.T.Horowitz and R.C.Myers, JHEP 0001:021 (2000). hep-th/9912135

A.Chamblin, C.Csaki, J.Erlich and T.J.Hollowood, Phys. Rev. D62 044012 (2000). hepth/0002076

R.Gregory, Black string instabilities in ADS space, hep-th/0004101.

[42] A.Ishibashi and H.Ishihara, Phys. Rev. D56 3446 (1997). [gr-qc/9704058

A.Ishibashi and H.Ishihara, Phys. Rev. D60 124016 (1999). [gr-qc/9802036]

[43] F.Bonjour, C.Charmousis and R.Gregory, The dynamics of curved gravitating walls, gr-qc/0002063.

[44] A.Vilenkin, Phys. Rev. D41 3038 (1990).

B.Carter, Phys. Rev. D41 3869 (1990).

[45] S.Mukohyama, Gauge invariant gravitational perturbations of maximally symmetric spacetimes, hep-th/0004067.

H.Kodama, A.Ishibashi and O.Sato, Brane world cosmology - gauge invariant formalism for perturbation, hep-th/0004160.

[46] D.Langlois, Brane cosmological perturbations, hep-th/0005025.

C.van de Bruck, M.Dorca, R.H.Brandenberger and A.Lukas, Cosmological perturbations in brane world theories: formalism, hep-th/0005032.

K.Koyama and J.Soda, Evolution of cosmological perturbations in the brane world, hepth/0005239. 